\title{
Avaliação do crescimento de crianças: a trajetória das curvas de crescimento
}

\section{Evaluation of the growth of children: path of the growth charts}

\author{
Aline Alves Ferreira ${ }^{1}$ \\ ' Escola Nacional de Saúde Pública Sérgio \\ Arouca, Fundação Oswaldo Cruz (ENSP/Fiocruz) \\ Instituto de Nutrição, Universidade do Estado do \\ Rio de Janeiro (INU/ UERJ). \\ Correspondência / Correspondence \\ Aline Alves Ferreira \\ E-mail: aline_af@yahoo.com.br
}

\section{Resumo}

O objetivo do artigo é apresentar e discutir, a partir da revisão de literatura, conceitos relacionados às curvas de crescimento infantil, ressaltando aspectos históricos, metodológicos e antropométricos. Utilizaram-se as bases de dados PubMed, SciELO e Scopus. Foram incluídos manuscritos, livros técnicos, sites de organizações e órgãos nacionais e internacionais que abordavam o tema nos idiomas português, inglês e espanhol, no período de 1966 a 2012. Passando desde Guarinoni, Montbeillard e outros estudiosos, o conceito de crescimento infantil ganhou contornos mais populares, principalmente no que se referia à maneira de quantificá-lo e avaliá-lo através das curvas de crescimento. Diversas foram as etapas para se chegar à atual "curva ideal de crescimento infantil", atualmente representada pelas referências e padrões de crescimento propostos pela OMS em 2006 e 2007. Apesar de sua ampla difusão entre países, releituras, críticas e readaptações dessas curvas envolvendo especificidades locais e de idade estão sendo realizadas. Diante dos novos complexos de problemas na saúde infantil, em um intervalo de tempo mais curto, pode ser gerada uma nova proposta de padrão e referência internacional. Paradoxalmente, parece que estamos caminhando a passos bem mais lentos em direção à especificidade, tendo cada grupo sua curva de crescimento infantil própria.

Palavras-chave: Gráficos de Crescimento. Crescimento. Criança. Antropometria. 


\section{Abstract}

This paper aims to present and discuss, from the literature review, concepts related to child growth charts, emphasizing historical, methodological and anthropometric aspects. Study databases comprised PubMed, Scopus and SciELO. Manuscripts, technical books, websites, organizations and national and international organs that addressed this topic in Portuguese, English and Spanish, in the period from 1966 to 2012, were also included. From Guarinoni, Montbeillard and other researchers, the concept of child growth gained more popular contours, especially when it came to how to quantify it and evaluate it through growth curves. Several steps were taken to arrive at current "optimal child growth curve", currently represented by references and growth patterns proposed by WHO in 2006 and 2007. Despite its widespread diffusion among countries and readings, there are critiques and re-adaptations of these curves involving local specificities and age. Faced with the new complex problems in child health in a shorter time can be generated a new proposal for standard and international reference. Paradoxically, it seems that weo go little by little towards specificity: each group with its own children's growth curve.

Key words: Growth Charts. Growth. Child. Anthropometry.

\section{Introdução}

O crescimento somático é um processo complexo, flexível e, portanto, variável. ${ }^{1.2}$ Em crianças, é considerado um dos melhores indicadores de saúde e nutrição, refletindo a interação de inúmeros fatores intrínsecos e extrínsecos. ${ }^{3}$

A antropometria e o uso de tabelas e figuras são recursos comumente utilizados para avaliar o crescimento infantil. Esse processo pode ser resumido de forma gráfica a partir de distribuições de percentis (ou escores z) de valores de medidas antropométricas de crianças consideradas referências. Assim, são construídas as chamadas curvas ou gráficos de crescimento. ${ }^{3,4}$

Tais ferramentas são utilizadas por diferentes profissionais de saúde e consideradas fundamentais para a avaliação e monitoramento do estado de saúde da criança, tanto na clínica quanto na saúde pública. ${ }^{3-5}$ No entanto, a construção dessas curvas e a busca pela melhor população que representasse o crescimento das crianças passou por diversas etapas até os dias atuais.

Nesse contexto, o objetivo do presente estudo é apresentar e discutir, a partir da revisão de literatura, conceitos relacionados às curvas de crescimento infantil, ressaltando aspectos históricos, metodológicos e antropométricos, sob a ótica do crescimento físico de crianças. 


\section{Fontes dos dados}

Este artigo se baseia nos principais textos que influenciaram os conceitos atuais sobre a avaliação do crescimento e curvas de crescimento de crianças. Foi realizada pesquisa bibliográfica entre agosto de 2011 e junho de 2012, utilizando os idiomas português, espanhol e inglês. Foram consultadas as bases de dados PubMed, SciELO e Scopus, utilizando-se a combinação dos descritores e palavraschave: growth charts, children, childhood, growth, body weight, body height, child development e growth references e assessment of growth. O Portal Capes também foi acessado para consultas.

Com base na leitura dos resumos, foram excluídos os que não se encaixavam na temática, perfazendo um total de 47 artigos e nove livros. Destes, foram selecionados 34 , que condiziam melhor com o tema do presente estudo. Pesquisou-se também em acervos de bibliotecas os livros técnicos sobre o tema, além de sites de organizações e órgãos nacionais e internacionais. Foram consideradas as publicações dos períodos de 1966 a 2012.

\section{Uma perspectiva histórica do crescimento físico e construção das curvas de crescimento de crianças}

Diferentes vertentes e focos impulsionaram os estudos sobre crescimento humano nos últimos séculos, consequentemente influenciando a construção das curvas de crescimento infantil. Um dos estudos mais rudimentares sobre crescimento de crianças foi no século XVI. Hippolyt Guarinoni associou o retardo no crescimento de meninos a causas emocionais, oriundos de períodos de estresses na escola. ${ }^{3}$

No entanto, o primeiro estudo que abordou o tema de forma sistemática e longitudinal foi o de Philibert Guéneau de Montbeillard, na França. Ele analisou o crescimento de seu filho do nascimento (1759) até 1777, na forma gráfica e em tabelas, estabelecendo a existência do esporão de crescimento puberal e mudanças sazonais na taxa de crescimento. Confirmou também a ocorrência de "encolhimento" estatural durante o dia. Pode ser considerado um trabalho bastante refinado para época, com boa precisão, ainda que não tenha análises estatísticas robustas, nem o emprego de técnicas de amostragem. ${ }^{3,6}$

Na Europa, no início do século XIX, juntamente com a Revolução Industrial, surgiu uma nova linha de estudos sobre crescimento humano. Eram investigações originárias das reações de correntes humanitárias diante das condições de pobreza dos trabalhadores e seus filhos. Os dados sobre crescimento passaram a ser utilizados para definir condições de saúde ideais e as consideradas precárias, abaixo do ideal mínimo. Inquéritos de saúde com crianças em idade escolar e tentativas de monitoramento do estado nutricional começaram a ser realizados. ${ }^{6}$ 
Para a Nutrição em Saúde Pública, Adolphe Quetelet foi uma das figuras mais importantes. É considerado por alguns como o pai dos estudos na era moderna sobre o crescimento humano e fundador da estatística moderna, introduzindo na prática a aplicação da curva normal. Foi o primeiro a realizar um inquérito populacional (transversal) com crianças, nos anos de 1831 e 1832, empregando técnicas modernas de estatísticas para a época e associando os diferentes padrões de crescimento a fatores ambientais. Influenciado por Louis-René Villermé, que dois anos antes havia publicado um trabalho abordando os efeitos da pobreza sobre a altura de recrutas franceses, a pesquisa de Quetelet culminou na publicação mais importante sobre padrões de crescimento em crianças: Sur l'homme et le développement de ses facultés. Também foi o responsável pela criação de índice amplamente empregado nos dias de hoje, relacionando peso e altura - índice de massa corporal (IMC) ou índice de Quetelet. ${ }^{6}$

Paralelamente, a antropometria vinha se consolidando como método em estudos sobre crescimento de crianças no campo da saúde pública, sendo incorporada na prática médica. A necessidade de compração a partir dos dados coligidos dos paciente começou a surgir. Assim, dados considerados referências começaram a ser coletados de forma mais séria e sistemática a partir do século XIX,${ }^{3}$ surgindo, ainda no final desse século, os primeiros gráficos de crescimento. ${ }^{1,6}$

A partir dos estudos de James Mourilyan Tanner, no final do século XIX e início século XX, e da criação do Serviço de Crescimento Humano na Inglaterra, a análise do crescimento passou a ter caráter mais amplo, sendo definida por um conjunto de fenômenos celulares, físico-químicos, morfológicos e genéticos. $\mathrm{O}$ ambiente passou a ser considerado importante fator modificador. O conceito de crescimento ganhou contornos mais populares entre os estudiosos da época, principalmente no que se referia à maneira de quantificá-lo e avaliá-lo. Difundiam-se, assim, as referências de velocidade e gráficos de crescimento., ${ }^{3,7}$

\section{0 caminho até as atuais referências e padrões de crescimento infantil}

A precisão na avaliação do crescimento tem sido do interesse de diversas áreas da Saúde Pública e Biologia humana há décadas. Através da antropometria, método mais comumente utilizado na avaliação do estado nutricional, ${ }^{4}$ são obtidos dados que podem ser comparados com os dados de populações considerados referências, ou seja, indivíduos sãos, que vivem em condições socioambientais e econômicas ideias para um bom desenvolvimento. A síntese dos valores antropométricos desses indivíduos constituem uma referência de crescimento, expresso de forma gráfica (curvas de crescimento) ou em tabelas. ${ }^{8}$

As curvas de crescimento são construídas a partir de dados longitudinais ou transversias. Mostram a relação entre variáveis antropométricas e demográficas, como por exemplo, peso e idade, através de uma curva única resumida, expressa em percentis ou escores z. O gráfico é 
representado por uma ou mais linhas que crescem progressivamente, com incrementos gradativos de medidas antropométricas, de acordo com sexo e idade, desde o nascimento. ${ }^{8}$

Podem ser considerados instrumentos técnicos versáteis e importantes na aferição, monitoramento e avaliação do crescimento de crianças, em nível individual ou coletivo. São bastante utilizadas na prática clínica, por diferentes profissionais de saúde, para monitorar individualmente a criança e também como ferramenta na saúde pública, para resumir e comparar antropometricamente os grupos de crianças. É considerado um indicador bastante sensível para a avaliação da saúde infantil como um todo, possibilitando intervenções específicas e prevenção de agravos à saúde. ${ }^{7,9}$

E qual população de referência deve ser utilizada na avaliação do crescimento infantil? Essa pergunta tem recebido atenção especial nas últimas décadas. As discussões acerca do desenvolvimento de curvas universais ou específicas para determinadas populações surgiram há mais tempo ainda. Independentemente do tipo de curva, o principal objetivo era construir um parâmetro que demonstrasse um ideal de crescimento. ${ }^{3,7}$

Alguns autores defendiam a construção de curvas de crescimento locais, a partir de referências nacionais ou específicas para determinados grupos. Alegavam que havia forte influência dos fatores genéticos e étnicos no desenvolvimento e crescimento das crianças, inclusive nos primeiros anos de vida. ${ }^{2}$ Já outros defendiam que esses fatores, até o quinto ano de vida, eram menos importantes que as características ambientais nas quais a criança estava submetida. Logo, o crescimento até essa idade seria universal em qualquer população que pudesse ser considerada saudável. ${ }^{9}$

Partindo da premissa de que as curvas originárias de populações consideradas referências poderiam ser satisfatoriamente aplicadas a todos as crianças do mundo, caminhou-se para a construção de uma única curva de crescimento infantil. ${ }^{9}$ Inúmeras foram as tentativas de construção de curvas voltadas para uso internacional. Umas das primeiras foram as curvas de Iowa, Estados Unidos. A partir de estudo longitudinal iniciado na primeira metade do século XX (Iowa Child Welfare Study), com crianças da cor branca, foram construídas as curvas de crescimento. Em pouco tempo, esses dados não se mostraram universais e satisfatórios para serem aplicados em outras populações. ${ }^{10}$

O primeiro gráfico de referência mais utilizado no âmbito internacional e recomendado pela OMS foi a curva de Harvard (EUA). Constituída a partir de estudos transversais (entre 19301956) com crianças caucasianas norte-americanas de Boston, continha os índices peso-para-idade, estatura-para-idade e peso-para-estatura para menores de 36 meses de ambos os sexos, além dos dois primeiros índices para jovens entre dois e 18 anos. Foi bastante utilizada pelos pediatras da época e sua maior crítica derivava do fato de a amostra não ser nacionalmente representativa. ${ }^{8,10}$

Um padrão de crescimento, a classificação de Gómez, era mais disseminado no contexto internacional que as curvas de Harvard, principalmente nos países pan-americanos. ${ }^{10}$ Vale ressaltar 
que, em termos teóricos, há importante diferença entre os termos "padrão" e "referência”. O primeiro abrange a noção de uma norma ou meta desejável, envolvendo um juízo de valor. Já o segundo é definido como uma ferramenta para o agrupamento e análise de dados, fornecendo uma base comum para comparar populações, sem inferências sobre os significados das diferenças observadas. Os dados de referência podem incorporar certas características ou padrões de normalidade e, com isso, serem utilizados para fazer inferências sobre a saúde e/ou nutrição de indivíduos e populações. ${ }^{3,10} \mathrm{O}$ padrão de crescimento de Gómez influenciou inúmeras tentativas não apenas de padronização, mas de construção de curvas de crescimento locais em países menos desenvolvidos, inclusive no Brasil. Nas décadas de 1960 e 1970, Eduardo Marcondes desenvolveu curvas de crescimento no país através de dois estudos transversais, com indivíduos de 0 a 20 anos, de elevado status socioeconômico da cidade de Santo André. ${ }^{11}$ Além da utilização de metodologias não-robustas, não possuía validação externa e não era representativa da população brasileira. ${ }^{8}$

No mesmo período, Tanner et al. ${ }^{12}$ elaboraram curvas voltadas para a velocidade de crescimento, a partir de amostra de crianças britânicas do Centro de Estudos da Criança (atual Centro para o Estudo do Desenvolvimento Humano), seguidas desde o nascimento. O conceito de velocidade aplicado graficamente era inédito para a época, mas por motivos semelhantes à metodologia das curvas de Harvard, não foi difundido internacionalmente. ${ }^{10}$

No final dos anos 1970, a OMS divulgou uma referência com representatividade nacional e que, mais tarde, foi aceita também como novo padrão de crescimento: a curva do National Center for Health Statistics - NCHS. ${ }^{10}$ Desde então, vários países passaram a utilizar a mesma referência, possibilitando comparações entre várias regiões do mundo. A curva do NCHS foi construída a partir de dois estudos longitudinais na população norte-americana ${ }^{13,14}$ : : um com crianças menores de dois anos (entre 1929 e 1975); e outro para as demais, indo até 18 anos (entre 1960 e 1975). Uma das limitações era sua utilização em adolescentes (maiores de 10 anos): as curvas do NCHS abrangiam apenas os 18 anos e não os menores de 20, como é preconizado o período da adolescência. Além disso, o tamanho amostral oscilou em diferentes idades e não foram levadas em consideração as diferenças nas idades médias de ocorrência da puberdade nos vários grupos étnicos norte-americanos. ${ }^{5}$

A partir do final dos anos 1980, críticas mais contundentes em relação aos limites de percentis utilizados e procedimentos estatísticos inapropriados para descrever o padrão e a variabilidade crescimento começaram a surgir. Assim, em 2000, o NCHS publicou uma nova referência do Center for Disease Control and Prevention (CDC), incluindo na amostra 14\% de jovens negros. Os dados eram provenientes de cinco estudos realizados entre 1963 e 1994. Além de distintos índices para todas as faixas de idade, incorporou-se também o IMC-para-idade. ${ }^{15}$ No entanto, não incluíram crianças nascidas com baixo peso, não necessariamente com aleitamento materno exclusivo e a população baseline continuava a ser dos Estados Unidos. ${ }^{16}$ 
Após abrangente revisão do uso e interpretação das curvas propostas pelo NCHS, em 1993, especialistas concluíram que essa referência não era adequada para avaliação e monitoramento do crescimento de crianças em todas as partes do mundo. Iniciaram-se então planejamentos e ações que culminaram nas chamadas novas curvas de crescimento da OMS, publicadas em 2006 e $2007.17,18$

\section{Novas curvas da OMS: apenas a recomendação atual ou chegamos a um consenso?}

Recomenda-se atualmente o uso dos padrões e referências internacionais de crescimento publicadas pela OMS em 2006 e 2007. Mais de 140 países, incluindo os menos favorecidos economicamente, estão em processo de implementação dessas curvas. ${ }^{19}$ Os gráficos da OMS apresentam importantes diferenças em relação às referências anteriormente utilizadas, principalmente do NCHS, ${ }^{13,14}$ A partir de uma equipe multidisciplinar, foi conduzido um estudo híbrido multicêntrico com mais de 8.500 jovens de diferentes grupos étnicos e nações que viviam em condições ambientais consideradas ótimas para o crescimento. Dentre os aspectos inovadores, incluem as curvas de velocidade de crescimento, a relação entre o desenvolvimento físico e motor e a definição do aleitamento materno como parâmetro para o crescimento ideal. Uma importante modificação foi a inclusão do IMC em relação à idade e a saída do índice peso-para-estatura da prática médica $^{17,18}$

As novas curvas conseguem detectar melhor as falhas no processo de crescimento, possibilitando intervenções precoces, além de serem mais sensíveis ao sobrepeso e obesidade, principal problema nutricional nos dias de hoje. É considerado um instrumento mais robusto em termos técnicos, tratando-se não apenas de uma referência, mas também de um novo padrão internacional. ${ }^{16,19}$

Aparentemente, as novas curvas de crescimento da OMS sanaram os principais problemas concernentes à metodologia das referências utilizadas anteriormente e têm sido recomendadas na avaliação do padrão ideal de crescimento de crianças e adolescentes. Sua aplicabilidade parte da premissa de uma baixa influência da variabilidade genética no potencial de crescimento infantil, quando comparadas à variabilidade ambiental. Assim, quando submetidas a condições ideais, crianças, independentemente de suas origens, crescem igualmente.

Devido à recente publicação, ainda há um processo de transição na utilização das novas curvas. Questiona-se sobre sua aplicabilidade internacional em determinados grupos, tais como os de estatura bem menores que o padrão usual, como os pigmeus na África Central, ou os Ianomâmis no Brasil. ${ }^{20}$ De acordo com Waterlow, ${ }^{20}$ existem diferenças genéticas inatas entre diferentes grupos étnicos, afetando diretamente a altura final dos indivíduos na fase adulta. Contudo, parece ser de opinião geral que hoje não existem diferenças significativas no potencial de crescimento entre diferentes grupos étnicos, ou se tais diferenças existem, elas são pequenas. 
A exclusiva utilização das novas curvas na avaliação do perfil nutricional em alguns grupos étnicos também é discutida. O principal ponto não seria sua validade, mas a dificuldade de comparação, especialmente pela impossibilidade de reanálises de alguns dados e comparabilidade de prevalências de desnutrição. ${ }^{21,22}$ São necessárias, ainda, reavaliações de resultados do estado nutricional de estudos anteriores, bem como explorar o uso e validade funcional das normas, principalmente para índices que não estavam disponíveis anteriormente. ${ }^{16}$

Algumas publicações, depois de 2006, têm apresentado comparações de referências nacionais com as referências de crescimento da OMS, nem sempre concordando com os novos padrões. É o exemplo de países como os África do Sul, Emirados Árabes, Polônia, Hong Kong, Irã, Noruega, Alemanha e Reino Unido. ${ }^{21,23-30}$. Em outros casos, como no Reino Unido, mesclou-se o uso das curvas da OMS com curvas locais, para certas fases da infância. ${ }^{14}$ Outros países, como China, Dinamarca, Bélgica, Tchecoslováquia, Bolívia e Noruega, manifestaram reservas para a utilização das novas curvas, pois investigações têm demonstrado que o crescimento das crianças nesses locais são significativamente diferentes dos parâmetros propostos pela OMS. Assim, eles decidiram continuar utilizando seus próprios gráficos de base populacional para a referência de crescimento infantil. ${ }^{19}$

Para alguns autores, atualizações regulares de referências locais e comparações com as curvas da OMS a partir de estudos multicêntricos nacionais poderiam assegurar importantes informações sobre o crescimento infantil local, além de maior especificidade para o diagnóstico do estado nutricional em determinados grupos étnicos. ${ }^{23,27,38}$ Outros justificam a criação de curvas de crescimento locais baseando-se no fato de que, mesmo as curvas da OMS tendo amostras representativas de seis continentes, essas não poderiam ser aplicadas a determinados países devido a diferenças culturais, socioeconômicas e influência de fatores genéticos. ${ }^{23,25-28,30}$ Indo além, alguns recomendam sua utilização apenas em crianças com aleitamento materno exclusivo nos primeiros meses de vida, haja vista as diferenças significativas dos parâmetros antropométricos entre a referência da OMS e outras. ${ }^{31}$

Por outro lado, é bastante evidenciado na literatura que o crescimento até os cinco anos de idade sofre baixa influência genética. ${ }^{3,5,7,8,10} \mathrm{O}$ uso de curvas locais não tem sido encorajado pelas principais organizações e instituições do mundo, sobretudo pelas dificuldades técnicas e políticas. Apresentam distintas limitações metodológicas e financeiras relacionadas à seleção da população referência representativa. Acredita-se também que curvas construídas a partir de populações de países menos desenvolvidos, que são expostas a condições precárias de saúde e nutrição, teriam um valor de triagem menor na verificação da desnutrição. 8,10,19 $^{8}$ 
A busca pela chamada "curva ideal de crescimento infantil" pode ter sido minimizada periodicamente, com a divulgação das referências da OMS em 2006 e 2007, ainda que haja releituras, críticas e readaptações dessas curvas, envolvendo especificidades locais de alguns países. Apesar disso, nenhuma crítica mais contundente ainda foi gerada, sendo reconhecida, portanto, a relevância das novas curvas, especialmente à luz da utilização no cenário internacional.

\section{0 futuro}

Novos complexos de problemas surgiram ao longo do final do século passado. Distintos tipos de estresses ambientais, incluindo doenças (re)emergentes, alterações nos padrões alimentares e novos estilos de vida, causaram impactos importantes no crescimento de crianças. Novas demandas e questionamentos envolvendo estudos populacionais surgiram, tanto em níveis locais, quanto internacionais.

As pesquisas envolvendo crescimento e desenvolvimento infantil estão em fase de transição do descritivo para o analítico. Uma descrição populacional por si só perdeu o sentido. A necessidade de $p$-valores significativos a partir de inferências causais é uma realidade. Embora muitas das descrições sejam adequadas e necessárias para certos tipos de intervenções de saúde pública, existem muitas incertezas, sobretudo no entendimento do processo saúde-doença associado ao crescimento de crianças. Assim, cada vez mais busca-se a compreensão e, principalmente, os mecanismos associados ao crescimento das crianças em diferentes níveis: endócrino, imunológico, socioeconômico e genético.

Por mais necessárias que estratégias analíticas sejam incorporadas ao crescimento infantil, o instrumento mais consolidado, versátil, de mais baixo custo e rápido diagnóstico que possuímos atualmente são os gráficos de crescimento. Mesmo com limitações inerentes, esses fornecem um conjunto único de referências que permitem comparações de dados de diferentes grupos em várias partes do mundo. As populações que são e ainda serão utilizadas como base para a construção desses gráficos continuarão a ser questionadas.

Com a emergência e a necessidade de estudos longitudinais para a saúde pública, em um intervalo de tempo mais curto que as curvas do NCHS e da OMS, pode ser gerada uma nova proposta de padrão e referência internacional. Curvas mais específicas para as várias fases da infância já estão sendo desenvolvidas, ${ }^{31}$ de acordo com o peso ao nascer ${ }^{32}$ e o crescimento intrauterino. ${ }^{33}$ Assim, paradoxalmente à demanda de um único instrumento internacional, caminhamos a passos pouco mais lentos e discrepantes para a especificidade: cada país com sua curva de crescimento infantil própria. 


\section{Considerações finais}

O crescimento físico na infância é um dos eventos mais complexos que ocorrem ao longo da vida humana. A avaliação quantitativa desse processo foi alvo de inúmeros estudiosos ao longos dos séculos, e a antropometria, por suas facilidades e relativa precisão, tem-se consolidado como um dos métodos mais importante na avaliação do crescimento.

Nos últimos 200 anos, os gráficos de crescimento desenvolveram-se consideravelmente. Eles representam uma síntese notória do crescimento físico de crianças. A influência do meio em que a criança vive parece ser maior que a influência genética e étnica. Assim, caminhou-se, entre várias tentativas, para a construção de um referencial de crescimento internacional.

Hoje em dia as curvas de crescimento da OMS são recomendadas para serem utilizadas em quaisquer sociedades, independentemente de sua origem étnica e condição socioeconômica. Dentre seus aspectos inovadores, destaca-se o fato se serem oriundas de um estudo multicêntrico, com uma amostra de crianças que tiveram aleitamento materno exclusivo. Apresentam também curvas de velocidade de crescimento, além da relação entre aspectos do desenvolvimento físico e motor.

As novas curvas da OMS têm sido de grande utilidade, fornecendo um único conjunto de referências e padrões de crescimento que permitam comparações de dados de diferentes populações. No entanto, testes e comparaçãos com populações locais e grupos étnicos específicos têm sido evidenciados na literatura. Muitos países adotaram as novas curvas, mas alguns têm indagado sua utilização, propondo inclusive a utilização de referências locais. Questionamentos sobre a amostra também começaram a aparecer. A atualização desses dados amostrais será imprescindível com o passar do tempo, principalmente se analisado à luz das rápidas mudanças no estilo de vida moderno, no processo saúde-doença e no padrão de alimentação dessas crianças. Contudo, por todas as vantagens já apresentadas e por todo o cuidado metodológico envolvido em sua construção e análise, as curvas de crescimento para crianças da OMS são as melhores que temos atualmente. E talvez as melhores que teremos por algum tempo.

\section{Referências}

1. Bogin B. Patterns of Human Growth. Cambridge: Cambridge University Press; 1988.

2. Tanner JM. Fetus into man: physical growth from conception to maturity. Revised ed. Cambridge: Harvard University Press; 1989.

3. Ulijaszek SJ, Johnston FE, Preece MA. The Cambridge encyclopedia of human growth and development. Cambridge: Cambridge University Press; 1998.

4. Gibson RS. Principles of Nutritional Assessment. Oxford: Oxford University Press; 1990. 
5. World Health Organization. Physical status: the use and interpretation of anthropometry. Report of a WHO Expert Committee. World Health Organ Tech Rep Ser. 1995;(854):1-452.

6. Tanner JM. A history of the study of human growth. Cambridge: Cambridge University Press; 1981.

7. Eveleth PB, Tanner JM. Worldwide variation in human growth. Cambridge: Cambridge University Press; 1990.

8. Onis M, Habitch JP. Anthropometric reference data for international use: recommendations from a World Health Organization Expert Committee. Am J Clin Nutr. 1996;(64):650-8.

9. Habicht JP et al. Height and weight standards for preschool children: how relevant are ethinic differences in growth potential? Lancet. 1974;1(7.858):611-4.

10. Onis M, Yip R. The WHO growth chart: historical considerations and current scientific issues. In: Porrini M, Walter P, Editors. Nutrition in Pregnancy and Growth. Bibl Nutr Dieta. 1996;(53):74-89.

11. Marcondes E. Normas para o diagnóstico e a classificação dos distúrbios do crescimento e da nutrição - última versão. Pediatria (São Paulo). 1982;(4):307-26.

12. Tanner JM, Whitehouse RH, Takaishi M. Standards from Birth to Maturity for Height, Weight, Height Velocity, and Weight Velocity: British Children, I965 - Part I and II. Arch Dis Child. 1966;41(454):1-18.

13. Hamill PV et al. NCHS growth curves for children birth-18 years: United States.Vital Health Stat. 1977;(165):1-74.

14. Hamill PV et al. Physical growth: National Center for Health Statistics percentiles.Am J Clin Nutr. 1979;32(3):607-29.

15. Kuczmarski RJ et al. CDC growth charts for the United States: methods and development. Vital Health Stat., 2002;(246):1-190.

16. Van Den Broeck J, Willie D, Younger N. The World Health Organization child growth standards: expected implications for clinical and epidemiological research. Eur J Pediatr. 2009;168(2):247-51.

17. World Health Organization. WHO child growth standards: methods and development. Length/ height-for-age, weight-for-age, weight-for-length, weight-for-height and body mass index-for-age. 2006 [Acesso em 2012 abr. 20]. Disponível em: <http://www.who.int/entity/childgrowth/standards/ Technical_report.pdf>

18. Onis $\mathrm{M}$ et al. Development of a WHO growth reference for school-aged children and adolescents. Bull World Health Organ. 2007;(85):660-7.

19. Rao S, Simmer K. World Health Organization growth charts for monitoring the growth of Australian children: time to begin the debate. J Paediatr Child Health. 2012;48(2):E84-90.

20. Waterlow JC. Reflections on stunting. 2011 [acesso em 2011 set 02];(1):1-8. Disponível em: <http:// www.smith-gordon-publishing.com/pdf/Chapter-1-Reflections on-stunting-JC-Waterlow.pdf >

21. Norris SA et al. Implications of adopting the WHO 2006 Child Growth Standards: Case study from urban South Africa, the Birth to Twenty cohort. Ann Hum Biol. 2009;36(1):21-7.

22. Orellana JDY et al. Morbidade hospitalar em crianças indígenas Suruí menores de dez anos, Rondônia, Brasil: 2000 a 2004. Rev Bras Saude Mater Infant. 2007;7(3):281-7. 
23. Abdulrazzaq YM, Moussa MA, Nagelkerke N. National Growth Charts for the United Arab Emirates. J Epidemiol. 2008;18(6):295-303.

24. Cole TJ et al. Designing the new UK-WHO growth charts to enhance assessment of growth around birth. Arch Dis Child Fetal Neonatal Ed. 2012;97(3):F219-F222.

25. Heydari S, Emamghoreishi F, Amini M. Infants' Growth Charts in Jahrom, Iran. Iran J Pediatr. 2009;19(1):25-34.

26. Hui LL et al. Are universal standards for optimal infant growth appropriate? Evidence from a Hong Kong Chinese birth cohort. Arch Dis Child. 2008;93(7):561-5.

27. Júlíusson PB et al. Growth charts for Norwegian children. Tidsskr Nor Laegeforen. 2009;129(4):281-6.

28. Kułaga Z et al. Polish 2010 growth references for school-aged children and adolescents. Eur J Pediatr. 2011;170(5):599-609.

29. Wright $\mathrm{C}$ et al. Implications of adopting the WHO 2006 Child Growth Standard in the UK: two prospective cohort studies. Arch Dis Child. 2008;93:566-9.

30. Rosario AS, Schienkiewitz A, Neuhauser H. German height references for children aged 0 under 18 years compared to WHO and CDC growth charts. Ann Hum Biol. 2011;38(2):121-30.

31. Bertino E et al. Neonatal growth charts. J Matern Fetal Med. 2012;25(supl. 1):67-9.

32. Sherry B et al. Evaluation of and recommendations for growth references for very low birth weight ( $<$ or $=1500$ grams) infants in the United States. Pediatrics. 2003;111(4):750-8.

33. Brock RS, Falcão MC. Avaliação nutricional do recém-nascido: limitações dos métodos atuais e novas perspectivas. Rev paul pediatr. 2008;26(1):70-6.

Recebido: $13 / 9 / 2012$

Aprovado: 30/11/2012 